\title{
Viscous and thermodynamic behaviour of glass-forming organic liquids
}

\author{
$\mathrm{K} S \mathrm{DUBEY}$ and P RAMACHANDRARAO ${ }^{\dagger *}$ \\ Department of Applied Physics, 'School of Materials Science and Technology, Institute of \\ Technology, Banaras Hindu University, Varanasi 221005 , India \\ MS received 13 June 1991; revised 6 September 1991
}

\begin{abstract}
The free energy difference $(\Delta G)$ between an undercooled liquid and its corresponding equilibrium solid has been evaluated on the basis of a method involving Taylor series expansion of $\Delta G$ around its value at the equilibrium melting temperature. The resultant expression is shown to be capable of correctly estimating $\Delta G$ at temperatures as low as the glass transition temperature. The method is then enlarged to obtain the configurational entropy and used in conjunction with the Adam and Gibbs model to derive a novel expression for the viscosity of undercooled liquids. Most commonly used expressions for the temperature dependence of viscosity are shown to be approximations of the equation obtained in this study.
\end{abstract}

Keywords. Viscosity; undercooled liquids; Kauzmann temperature; configurational entropy.

\section{Introduction}

An accurate thermodynamic description of the undercooled liquid state is essential for understanding the viscous behaviour, glass-forming ability, nucleation propensity and crystal growth tendency in many liquids. Often, the metastability of the liquids precludes thermodynamic measurements below the equilibrium melting temperature $\left(T_{m}\right)$. Parameters like the free energy difference $(\Delta G)$ between the undercooled liquid and the corresponding equilibrium solid have so far been estimated by assuming some form of temperature dependence for the heat capacity difference $\left(\Delta C_{p}\right)$ between these phases (Turnbull 1950; Hoffman 1958; Jones and Chadwick 1971; Singh and Holz 1983). Models such as that due to Adam and Gibbs (1965) require a knowledge of the configurational entropy $\Delta S_{c}$ for an evaluation of the viscosity of an undercooled liquid. In this paper we demonstrate that a procedure recently proposed by Lele et al (1985) for estimating $\Delta G$ is well suited for the determination of many of these important properties of metastable liquids. Using this procedure and the Adam and Gibbs model, we derive a general expression for viscosity of an undercooled liquid that encompasses many other empirical relations as appropriate approximations.

\section{Free energy and entropy of undercooled liquids}

Following Lele et al (1985), we can expand the free energies of a liquid and the solid in equilibtiun with it at the melting point $\left(T_{m}\right)$ using Taylor series. The expansion extends the free energy versus temperature ( $G$ vs $T$ ) plot of each phase into their metastable regions. Considering the difference between the expressions for the liquid

\footnotetext{
* For correspondence
} 
and the solid yields $\Delta G$ in the form

$$
\Delta G=\Delta S_{m} \Delta T-\Delta C_{p}^{m} \frac{\Delta T^{2}}{\left(T_{m}+T\right)}+\frac{1}{2}\left[\frac{\partial \Delta C_{p}}{\partial T}\right]_{T_{m}} \frac{\Delta T^{3}}{T_{m}+T}-, \ldots
$$

where $\Delta C_{p}^{m}$ and $\Delta S_{m}$ are the specific beat and entropy differences between the liquid and the corresponding solid respectively at the melting temperature $T_{m} . \Delta T$ is the undercooling $T_{m}-T$. It may be noted that the first term in the above equation is the well-known Turnbull (1950) approximation for $\Delta G$. At large values of $\Delta T$ and $\Delta C_{p}^{m}$ large corrections need to be made to the Turnbull expression. However, very often it may not be necessary to consider more than two terms in (1) as the contribution of the higher terms is invariably negligible. We shall demonstrate that the corrections to the Turnbull approximation to (1) are significant at large undercoolings.

A similar Taylor series expansion can be carried out with respect to the entropy $(S)$ of both phases to get an expression for the entropy difference between the liquid and solid phases $(\Delta S)$ resulting in

$$
\begin{aligned}
\Delta S= & \Delta S_{m}-2 \Delta C_{p}^{m} \frac{\Delta T}{T_{m}+T}+\left[\frac{\partial \Delta C_{p}}{\partial T}\right]_{T_{m}} \cdot \frac{\Delta T^{2}}{T_{m}+T} \\
& -\frac{1}{4}\left[\frac{\partial^{2} \Delta C_{p}}{\partial T^{2}}\right]_{T_{m}} \cdot \frac{\Delta T^{3}}{\left(T_{m}+T\right)}+\ldots
\end{aligned}
$$

Kauzman (1948) pointed out the difficulties associated with a purely kinetic description of glass transition. One such problem is the likelihood of $\Delta S$ being negative at some low temperature. The paradox was resolved by Gibbs and DiMarzio (1958) when they considered glass transition as a truly second-order transition. $\Delta S$ goes to zero at an ideal glass transition temperature designated $T_{K}$. This temperature can be evaluated by setting (2) to zero at $T=T_{K}$. $T_{K}$ can thus be expressed in terms of $\Delta S_{m:} \Delta C_{p}^{m}$ and its temperature derivatives at $T_{m}$ as a solution to the transcendental equation of the form

$$
\begin{aligned}
& \Delta S_{m}-T_{m}\left[\frac{\partial \Delta C_{p}}{\partial T}\right]_{T_{m}}+\left\{\Delta C_{p}^{m}-T_{m}\left[\frac{\partial \Delta C_{p}}{\partial T}\right]_{T_{m}}\right\} \ln \left(T_{K} / T_{m}\right) \\
& +\left[\frac{\partial \Delta C_{p}}{\partial T}\right]_{T_{m}} \cdot T_{K}=0 .
\end{aligned}
$$

In the derivation of (1) to (3) it is assumed that

$$
\ln \left(T_{m} / T\right)=2 \Delta T /\left(T_{m}+T\right) \text {. }
$$

It may be noted that thigher order derivatives of $\Delta C_{p}$ with respect to $T$ at $T=T_{m}$ have been neglected due to their extremely small contributions. Under these approximations the above equation gives the value of $T_{K}$ as

$$
T_{K}=T_{m}-\left[\frac{\left(\Delta S_{m}+2 \Delta C_{p}^{m}\right) \pm\left\{\left(\Delta S_{m t}+2 \Delta C_{p}^{m}\right)^{2}-8 \Delta S_{m} \cdot T_{m}\left[\frac{\partial \Delta C_{p}}{\partial T}\right]_{T_{m}}\right\}^{1 / 2}}{2\left[\frac{\partial \Delta C_{p}}{\partial T}\right]_{T_{m}}}\right] .
$$


Neglecting the term $\left[\partial \Delta C_{p} / \partial T\right]_{T_{m}}$ in (5), $T_{K}$ is given by

$$
T_{K}=T_{m}\left[\frac{2 D-1}{2 D+1}\right]
$$

where $D=\Delta C_{p}^{m} / \Delta S_{m}$.

The assumption of $\Delta S=0$ at $T=T_{K}$ facilitates expression of $\Delta S$ in terms of only $T_{K}, \Delta C_{p}$ and its derivatives at $T_{m}$. Thus we have

$$
\Delta S=\Delta C_{\not p}^{m} \ln \left(T / T_{K}\right)-\left[\frac{\partial \Delta C_{p}}{\partial T}\right]_{T_{u}}\left[T_{m} \ln \left(T / T_{K}\right)-\Delta T_{K}\right]+\ldots,
$$

where $\Delta T_{K}=T_{m}-T_{K}$. Equation (6) can be used to estimate the viscosity of undercooled liquids using the Adam and Gibbs (1965) model.

\section{Viscosity of undercooled liquids}

According to the molecular-kinetic theory of Adam and Gibbs (1965), the viscosity of an undercooled liquid can be expressed as

$$
\eta=A \exp \left[C / T \Delta S_{c}\right]
$$

where $A$ and $C$ are constants and $\Delta S_{c}$, the configurational entropy is given by

$$
\Delta S_{c}=\int\left[C_{p} \text { (liquid) }-C_{p}(\text { glass })\right] \mathrm{d} \ln T \text {. }
$$

Often, the experimentally determined value of $C_{p}$ (glass) is very close to that of the crystal and exhibits similar temperature dependence (Wong and Angell 1976). Hence the expression for $\Delta S$ given by (6) can be used in place of $\Delta S_{v}$. Consideration of higher order derivatives of $\Delta C_{p}$ and use of (4) yield an expression for $\eta$ of the form

$$
\ln \eta=\ln A+\frac{B\left(l+T_{K} / T\right)}{\left(T-T_{K}\right)\left[1+A_{1}\left(T-T_{K}\right)+A_{2}\left(T-T_{K}\right)^{2}+A_{3}\left(T-T_{K}\right)^{3}+\ldots\right]} .
$$

In case $\Delta C_{p}$ varies linearly with temperature

where

$$
\ln \eta=\ln A+\frac{C}{2 \Delta C_{p}^{m}} \frac{1}{\left(T-T_{K}\right)}\left(1+\frac{T_{K}}{T}\right) \frac{1}{\left\{1-\frac{\partial \Delta T_{K}}{T_{m}}\left(1-\frac{T-T_{K}}{2 \Delta T_{K}}\right)\right\}}
$$

$$
x=T_{m} \frac{\partial}{\partial T}\left[\ln \Delta C_{p}\right]_{T_{m}} \text { and } \Delta T_{K}=T_{m}-T_{K},
$$

where $B=C / 2 \Delta C_{p}^{m}, A_{1}, A_{2}, A_{3}$, etc are constants and depend on $\Delta C_{p}^{m}$ and its derivatives with respect to $T$ at $T=T_{m}$ and $T_{K}$. When $\Delta C_{p}$ is independent of temperature (9) reduces to

$$
\ln \eta=\ln A+\frac{C^{\prime}}{2\left(T-T_{K}\right)}\left(1+\frac{T_{K}}{T}\right)
$$

with $C^{\prime \prime}=C / \Delta C_{F}^{m}$. In the vicinity of the glass transition temperature $T_{g}, T_{K} / T$ is 
approximately 0.75 (see 84 ) and (9) reduces to the frequently used Vogel-Fulcher equation (Vogel 1921; Fulcher 1925)

$$
\ln n=\ln \eta_{0}+\frac{C^{\prime \prime}}{\left(T-T_{0}\right)}
$$

with $T_{0}=T_{K}$ and $C^{\prime \prime}=0.875 C^{\prime}$.

In general, $C^{\prime \prime}$ of the Vogel-Fulcher equation (11) should be temperature-dependent at least to the extent of

$$
C=\frac{C}{2}\left[1+\frac{T_{K}}{T}\right]
$$

Our analysis shows that the Vogel-Fulcher equation is in fact an approximate expression arising from the more general equation of the form given by (9). The complexity of the viscous behaviour and consequently the curvature in the $\ln \eta$ vs 1/T plots will thus depend upon the temperature dependence of $\Delta C_{p}$. It should be stressed that $(9)$ derived by us has less number of adjustable parameters than in the case of the Yogel-Fulcher expression. All other constants (9) except $A$ and $B$ are derivable from thermodynamic data and are not adjustable. They are dependent on $\Delta C_{t}$ and its temperalure dependence, Further, (9) with a single set of constants $A$ and $B$ should be applicable over the enlire temperature range of interest. Equation (9) of our analysis is to be preferred when $\Delta C_{p}$ is a strong function of $\Delta T$ and should have a definite advantage over the Vogel-Fulcher equation.

We shall now test (1) for $\Delta G$ and (9a) for viscosity on five well-studied organic glass-forming liquids. In each case the experimentaily determined $\Delta C_{p}$ can be expressed by

$$
\Delta C_{p}=a+b T
$$

and the experimental value of $\Delta G$ can be evaluated by integrating

$$
\left.\Delta G=\left[\Delta H_{m}-\int_{T}^{T_{m}} \Delta C_{p} \mathrm{~d} T\right]-T\left(\Delta S_{\mathrm{m}}-\int_{T}^{T_{m}} \Delta C_{p} \mathrm{~d} \mathrm{ln} T\right)\right] .
$$

\begin{tabular}{|c|c|c|c|c|c|}
\hline \multirow[b]{2}{*}{ Mateprial } & \multirow[b]{2}{*}{$\begin{array}{l}T_{m} \\
(\mathbf{K})\end{array}$} & \multirow[b]{2}{*}{$\begin{array}{l}T_{g} \\
(\mathrm{~K})\end{array}$} & \multirow[b]{2}{*}{$\underset{\mathrm{J} / \mathrm{mol} / \mathrm{K})}{\Delta S_{n}}$} & \multicolumn{2}{|c|}{ Constants in (12) } \\
\hline & & & & $\begin{array}{c}a \\
(\mathrm{~J} / \mathrm{mol} / \mathrm{K})\end{array}$ & $\begin{array}{c}b \\
\left(\mathrm{~J} / \mathrm{mol}^{\prime} \mathrm{K}^{2}\right)\end{array}$ \\
\hline Glycerol & 293 & 186 & $62 \cdot 70$ & 908646 & $-3906 \times 10^{-2}$ \\
\hline Ethanot & 158.5 & 91 & 31.64 & $51-5810$ & $-1.673 \times 10^{-1}$ \\
\hline 2-Methyl pentans & 119.55 & $79 \cdot 5$ & 5236 & 146.8710 & $-7.985 \times 10^{-1}$ \\
\hline o-terphenyl & .328 & $243 \cdot 15$ & 5601 & $241 \cdot 1280$ & $-4.933 \times 10^{-1}$ \\
\hline $\begin{array}{c}\text { 1,3,5-Tri-z- } \\
\text { naphthyl } \\
\text { bonzenc }\end{array}$ & 472 & 342 & 9002 & $311+4250$ & $-4673 \times 10^{-1}$ \\
\hline
\end{tabular}

The materials and their constants are shown in table 1.

Table 1. Parameters used lor the evaluation of $\Delta G$ of the materials under study.

(Kefly 1929; Parks et al 1930; Douslin and Hutimann 1946; Wuaderlich 1960; Magill 1967; Angell and Rav 1972) 


\section{Results and discussion}

Table 2 shows the $\Delta G$ values obtained from (1) and compares the same with the values calculated from (13) and shown as the experimental values. For the sake of brevity these are evaluated at normalized undercooled temperature $\left(T / T_{m}\right)$. In all cases, the agreement berween the calculated and experimental values of $\Delta G$ is excellent. Even at the glass transition temperature $T_{g}$, the difference is always less than $4 \%$ of the value obtained from (13). A comparison of the present results with those arrived at by the use of several expressions in current use [(14)-(17)] clearly brings out the utility and accuracy of (1). In table 3 are compared the $\Delta G$ values for glycerol estimated from (1) and the following expressions at various temperature from $T_{m}$ to $T_{g}$

$$
\begin{aligned}
& \Delta G=\Delta S_{m} \cdot \Delta T \text { (Turnbull 1950), } \\
& \Delta G=\Delta S_{m} \cdot \Delta T \cdot \frac{T}{T_{m}} \text { (Hoffman 1958), } \\
& \Delta G=\Delta S_{m} \cdot \Delta T \frac{2 T}{T_{m}+T} \text { (Thompson and Spaepen 1979) } \\
& \Delta G=\Delta S_{m} \cdot \Delta T \frac{7 T}{T_{m}+6 T} \text { (Singh and Holz 1983). }
\end{aligned}
$$

In all cases the values derived from (1) are the closest to the experimental values (obtained from (13)). All other expressions result in considerable error at large undercoolings. Such errors are most detrimental in estimating quantities like nucleation frequency and growth rate of erystal in undercooled liquids. In view of this we strongly advocate the use of (1) which is derived from mathematical concepts without reference to any model. A temperature dependence of $\Delta C_{p}$ is, however, inherent in (1) and enters through the use of $\Delta C_{p}^{m}, \partial \Delta C_{p} / \partial T$, etc. at $T_{m}$. It also enters

\begin{tabular}{|c|c|c|c|c|c|c|c|c|c|c|}
\hline \multirow[b]{3}{*}{$T T_{m}$} & \multicolumn{10}{|c|}{$\Delta G(\mathrm{~J} / \mathrm{mol})$} \\
\hline & \multicolumn{2}{|c|}{ Glycerol } & \multicolumn{2}{|c|}{$o$-terphenyl } & \multicolumn{2}{|c|}{$\begin{array}{c}1,3,5-\text { Tri- } \alpha- \\
\text { Nuphthyl benzene }\end{array}$} & \multicolumn{2}{|c|}{ 2-Methylpentane } & \multicolumn{2}{|c|}{ Ethanol } \\
\hline & Calc, & Exp. & Calc, & Exp. & Calc. & Exp. & Cale. & Exp. & Calc. & Exp. \\
\hline 095 & 889 & 889 & 883 & 884 & 2066 & 2068 & 305 & 305 & 246 & 246 \\
\hline 090 & 1713 & 1716 & 1686 & 1693 & 3996 & 4009 & 591 & 592 & 480 & 480 \\
\hline 0.85 & 2470 & 2478 & 2390 & 2415 & 5757 & 5802 & 854 & 859 & 700 & 703 \\
\hline 0.80 & 3150 & 3169 & 2977 & 3036 & 7313 & 1422 & 1090 & 1103 & 905 & 911 \\
\hline 0.75 & 3747 & 3786 & 3425 & 3543 & 8626 & 8843 & 1295 & 1320 & 1093 & 1105 \\
\hline 070 & 4253 & 4322 & - & - & - & - & 1462 & 1508 & 1261 & 1282 \\
\hline 0.65 & 4659 & 4772 & - & - & - & - & - & - & 1406 & 1440 \\
\hline$T_{g} / T_{m}$ & 4761 & 4890 & 3487 & 3619 & 9185 & 9479 & 1554 & 1619 & 1575 & 1640 \\
\hline
\end{tabular}
in the required form by the very nature of Taylor series expansion since the second

Table 2. Free energy difference between the liquid and equilibrium solid phase (1) of various materials. The experimental value was obtained by the use of (12). 


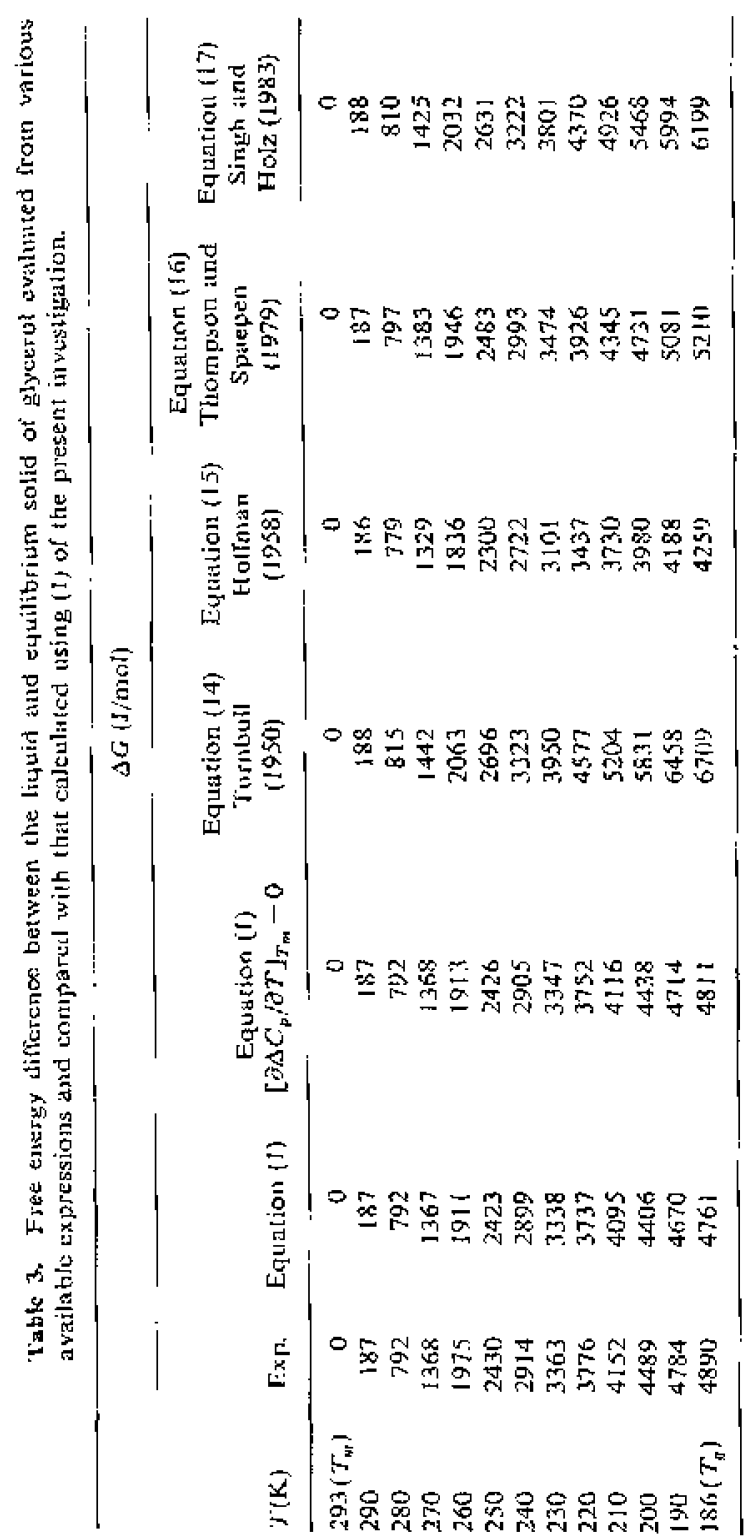


Table 4. Constants for use in (9a) for the viscosity of undercooled melts.

\begin{tabular}{|c|c|c|c|c|c|c|c|}
\hline Material & $T_{M}(\mathrm{~K})$ & $T_{K}(\mathrm{~K})$ & $A$ (poise) & $C$ (poise $K)$ & $x$ & $\begin{array}{l}\text { Viscosity } \\
\text { галge }\end{array}$ & $\begin{array}{c}\text { Correlation } \\
\text { (r) }\end{array}$ \\
\hline $\begin{array}{c}\text { 1,3,5-Tri-s } \\
\text { Nephthyl } \\
\text { benzene }\end{array}$ & 472 & $255 \cdot 4$ & $3.6392 \times 10^{-10}$ & 44847590 & $-2 \cdot 4273$ & $10^{t 3}-10^{-1}$ & 09989 \\
\hline a-terphenyl & 328 & $200 \cdot 2$ & $1.0088 \times 10^{-7}$ & 16924021 & $-2 \cdot 0353$ & $10^{13}-10^{-1}$ & $0-9977$ \\
\hline 2-Methy] pentane & $119 \cdot 55$ & $60 \cdot 9$ & $2.4049 \times 10^{-4}$ & $716 \cdot 0881$ & -1.8569 & $10^{11}-10^{4}$ & 0.9911 \\
\hline Giycerol & 293 & 137.5 & $3.6144 \times 10^{-4}$ & 5130195 & -0.1441 & $10^{4}-10^{0}$ & 0.9994 \\
\hline
\end{tabular}

and higher temperature derivatives of $\Delta G$ are related to $\Delta C_{p}$ and its temperature dependence.

The temperature dependence of the viscosity can also be arrived at from the $\Delta C_{p}$ data of table 1 in conjection with (9a). Towards this end we have estimated the Kauzmann temperature $T_{K}$ from $\Delta C_{p}$ data and (3). Experimentally measured viscosity values (Plazek and Magill 1966; Ling and Williard 1968; Langhlin and Uhlmann 1972; Cuckerman et al 1973; Weast 1988) were then plotted against a temperature function,

$$
f(T)=\frac{1+\left(T_{K} / T\right)}{\left(T-T_{K}\right)\left[1-\frac{x \Delta T_{K}}{T_{m}}\left\{1-\frac{T-T_{K}}{2 \Delta T_{K}}\right\}\right]}
$$

In each case, the Kauzmann temperature $T_{K}$ and the constants $A$ and $B$ derived from the best fit straight line are shown in table 4 together with the correlation coefficients (r) for the straight line. The calculated and observed viscosity values for three of the materials discussed are shown in figure 1 . As can be seen from the figure and the values of the correlation coefficients (table 4) (9a) with only two parameters is obeyed over a wide range of temperatures.

We have also noticed that there is a linear relationship between the reduced glass transition temperature $\left(T_{g} / T_{m}\right)$ and the reduced Kauzmann temperature $\left(T_{\boldsymbol{K}} / T_{m}\right)$ for all the materials studied here (figure 2). Analytically the straight line is represented by

$$
T_{\theta} / T_{m}=0.832\left(T_{K} / T_{m}^{\prime}\right)+0.25
$$

with a correlation of 09948 . Consequently

$$
T_{a}=0.832 T_{K}+0.25 T_{m} .
$$

As a rule of thumb, $T_{\eta} / T_{m}$ is about $1 / 2$. In the present instance, $T_{K} / T_{m}$ of materials studied varies from 0.6 to 0.4 and $(20)$ is still well obeyed.

\section{Conclusion}

Equation (1) derived by us for evaluating the $\Delta G$ of an undercooled liquid has been shown to yjeld correct estimates even at large undercoolings. The expression for the entropy difference $(\Delta S)$ between the undercooled liquid and the solid in thermodynamic equilibrium with it (2) can be used to determine the Kauzmann glass transition temperature and yields values which are in close agreement with values reported earlier 


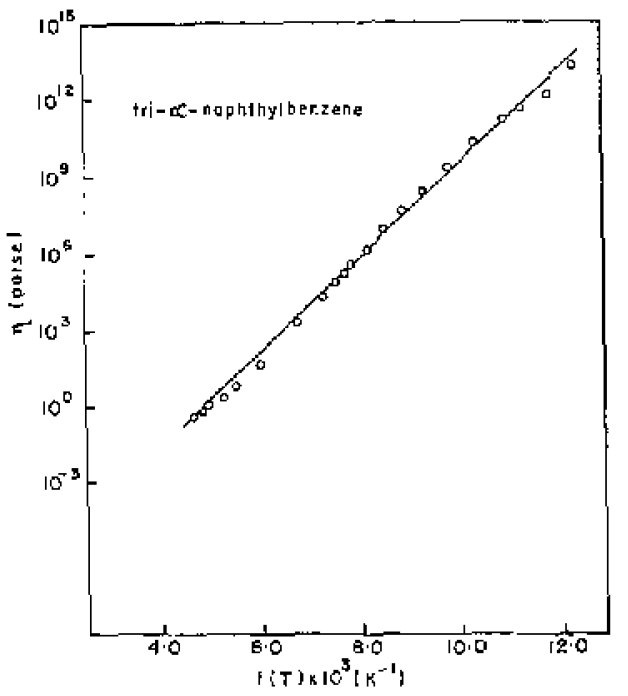

(a)

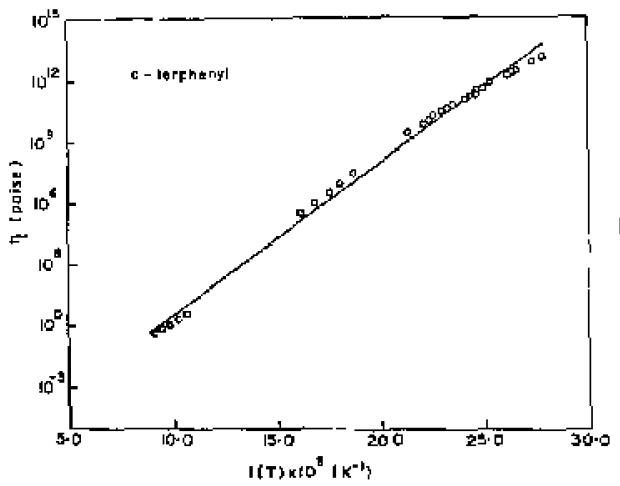

(b)

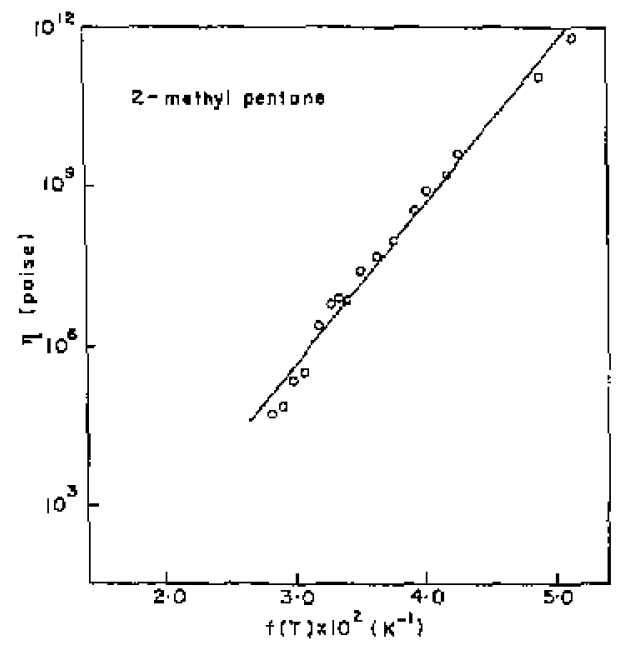

(c)

Figure 1. A comparison of the temperature dependence of the viscosity of undercooled liquids as predicted by equation (9a) (- - ) with the experimental values $(0)$. (a) $1,3,5-$ Tri- $^{-\alpha-}$ Naphthylbenzene (Plazek and Magill 1966), (b) o-terphenyl (Laughlin and Uhlmann 1972: Cuckerman et al 1973) and (c) 2-methylpentane (Ling and Williard 1968). 


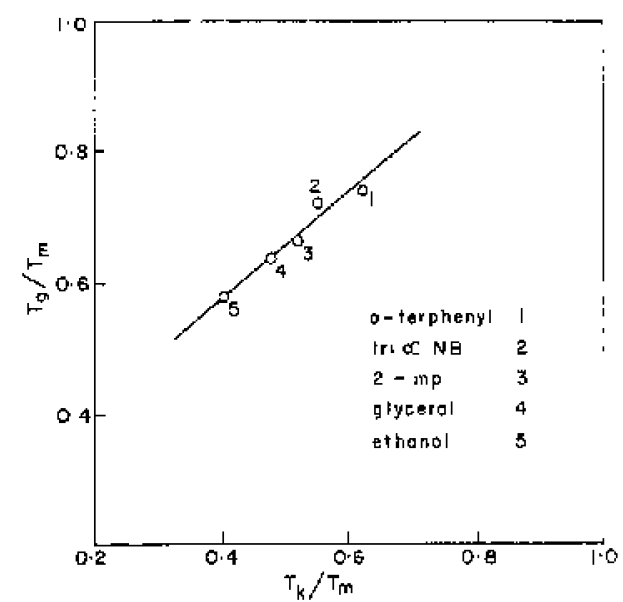

Figure 2. Linear telation between reduced $T_{p}$ and reduced $T_{K}$ for different glass forming liquids (equation (19)).

for a number of organic liquids. Both $\Delta G$ and $\Delta S$ are, as expected, strongly dependent on the temperature dependence of heat capacity and the values of the heat capacity and entropy difference ( $\Delta C_{p}^{m}$ and $\Delta S_{m}$ respectively) between the melt and the crystal at equilibrium melting temperature $\left(T_{m}\right)$. Both (1) and (2) require a knowledge of these parameters and the temperature dependence of $C_{p}^{L}$ and $C_{p}^{S}$ only in the temperature ranges where the two phases are stable i.e. for $T<T_{m}$ (near $T_{m}$ ) in the case of solid and $T>T_{m}$ (near $T_{m}$ ) in the case of liquid. Use of (2) in conjunction with the Adam and Gibbs model for viscous behaviour of undercooled liquids leads to a novel expression for the temperature dependence of viscosity of liquids. The new expression does not involve any more adjustable parameters than the Vogel-Fulcher equation but modifies the latter in a significant fashion. Applicability of our equation for viscosity with a single set of constants over a wide range of viscosity $\left(10^{-1}\right.$ to $10^{13} \mathrm{P}$ ) establishes its great utility.

\title{
References
}

\author{
Adam G and Gibbs J H 1965 J. Chem. Phys, 43139 \\ Angell C A and Rao K J 1972 J. Chem. Phys. 57470 \\ Cuckerman M, Lane J W and Uhlmann D R 1973 J. Chem. Phys, 593639 \\ Douslin D R and HuTmann H M $1946 \mathrm{~J}$. Am. Chem. Soc. 681704 \\ Fulcher G S $1925 \mathrm{~J}$. Am. Ceram. Soc. 773701 \\ Gibbs I H and DiMarzio E A 1958 J. Chem. Phys. 28373 \\ Holfman J D 1958 J. Chem. Phys. 291192 \\ Jones D R H and Chadwick G A 1971 Philos. Mag. 24995 \\ Kanino H 1981 J. Nom-Crystal Solids 44409 \\ Kauzman:n W 1948 Chem. Rev. 43219 \\ Kelly K K 1929 J. Am. Chem. Soc. $\mathbf{5 1} 779$ \\ Laughlin W T and Uhlmann D R 1972 J. Phys, Chem. 762317 \\ Lele S, Dubey K S and Ramachandrarao P 1985 Curr. Sci. 54994 \\ Ling $A C$ and Williard J E 1968 J. Phys. Chem. 721918 \\ Magill J H 1967 J. Chem. Phys. 472802 \\ Parks G S, Thomas S B and Gilley W A 1930 J. Phys. Chem. 342028
}


Plazek D J and Magill J H 1966 J. Chem. Phys, 453038

Singh H B and Holz. A 1983 Solid State Commum, 45985

Thompsen C V and Spaepen F 1979 Acta Metall. 271855

Tursbull D 1950 J. Appl. Phys. 211122

Vogel H 1921 Phis. Z. 22645

Weast R C 1988 CRC Handbook of chemistry and physses (Cleyeland: CRC Press)

Wong J and Angell C A 1976 Glass: Strtucture by spectroscopy (New York: Marcel Dekker)

Wunderlich B 1960 J. Fhys. Chem 64 1052 